\title{
Use the Frequency Domain to Hide in Some Audio Files
}

\section{Zahraa Tarik}

zahraata.eng@uomosul.edu.iq farahtarik@uomosul.edu.iq

College of engineering

University of Mosul

Received on: 15/10/ 2012
Farah T. Mohammd Ikhlass Thanoon Azhar Rafie College of computer science and mathematics

University of Mosul

Accepted on: 30/01/2013

\section{ABSTRACT}

In this work, anew hiding algorithm is proposed that depends on using two deferent domain (spatial and frequency) to achieve and provide security and protection for transformed data, which is represented in a text message encrypted using a key of direct standard method, then spatial domain were use to hide it inside an audio file, Using Discrete cosine transform (DCT) this file has been hidden inside a host audio file. The data of the text message has been fully retrieved after decrypting process, the value of normalization correlation is equal to one.

Key word: DCT, Audio, SNR, NC

$$
\begin{aligned}
& \text { استخدام المجال الترددي للإخفاء في بعض ملفات الصوت }
\end{aligned}
$$

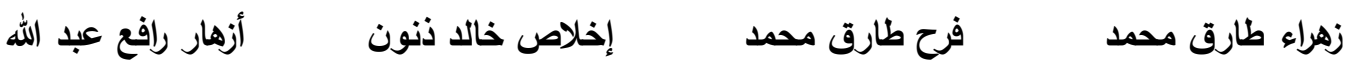

$$
\begin{aligned}
& \text { كلية علوم الحاسوب والرياضيات } \\
& \text { كلية الهندسة } \\
& \text { جامعة الدوصل } \\
& \text { جامعة الموصل }
\end{aligned}
$$

تاريخ قبول البحث: 2013/01/30

تاريخ استلام البحث: 2012/10/15

\section{الملخص}

تم في هذا العمل اقتراح خوارزمية تعتمد على استخدام مجالين مختلفين للإخفاء (المجال المكاني والمجال الترددي) لتحقيق وتوفير أمنية وحماية للبيانات المنقولة والمتمثلة في رسالة نصية مشفرة باستخدام مفتاح مدخل بطريقة وقد تم اعتماد المجال المكاني في لإخفائها داخل ملف صوتي وباستخدام تحويل الجيب تمام المتقطع (Discrete cosine transform (DCT) تَم إخفاء هذا الملف داخل ملف صوتي مضيف، وقد تم استرجاع بيانات الرسالة النصية بصورة كاملة بعد عملية فك الثفرة وكانت قيمة معامل الارتباط التعياري لها يساوي واحد. الكلمات المفتاحية: ملفات الصوت، تحويل الجيب تمام المتقطع.

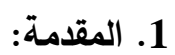

إن لإخفاء البيانات أهمية كبيرة للحفاظ على المعلومات الحقيقية وعدم ظهورها للعيان أياً كان نوعها ويعتبر

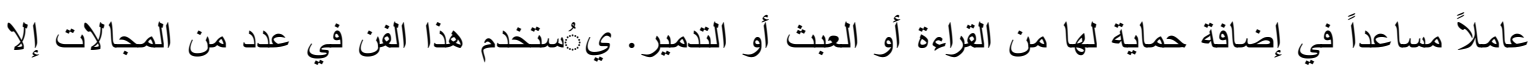

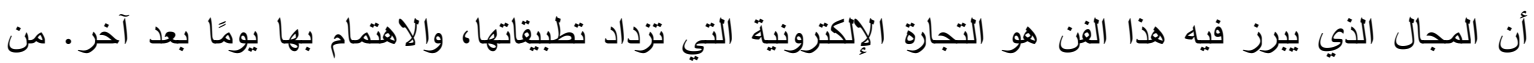

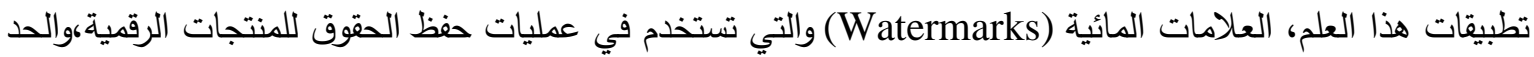

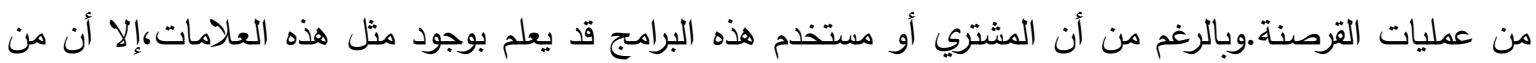

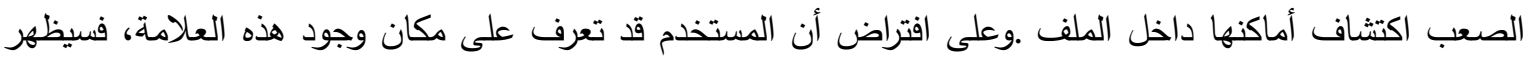

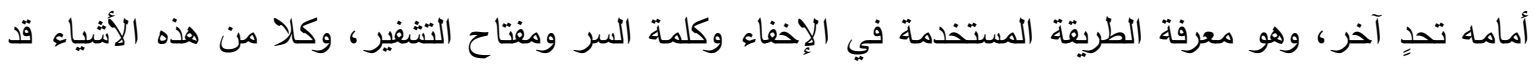

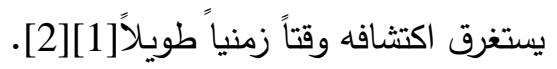


ومن الدراسات السابقة قام الباحث Jixin Lui باعتماد خوارزمية العلامة المائية الصوتية المتعدة الأغراض

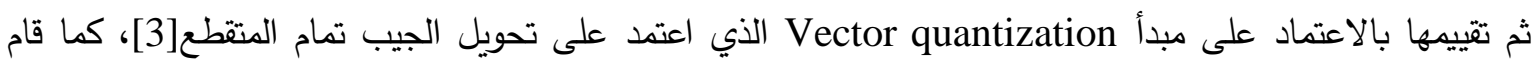
الباحثان Nidhi H Divecha و N Jani باقتراح خوارزمية تجمع بين مزايا التحويلات الثلاثة DWT، و و وSVD للصور الرقمية لتكون ضد كل أنواع الهجمات كون لديها قدرة عالية جدا على إخفاء البيانات[4].

يمكن اعتماد المجال المكاني والترددي في الإخفاء، حيث يمكن إخفاء بيانات النص المشفر داخل الملف الصوتي الأول باستخدام LSB فمثلاً لإخفاء حرف 'E' يتم تحويله إلى سلسلة من الأرقام الثنائية وبما أن كل sample ممثل بـbite لذا لذا يتم تغيير الـbit الأول أو الثاني حسب الخوارزمية ويعوض عنها بأحد أرقام السلسلة [5]. بعد تكوين الملف الوسطي يتم استخدام المجال الترددي باعتماد تحويل الجيب تمام المتقطع DCT للإخفاء في الملف الصوتي المضيف حيث يتم تقسيم الملف إلى مجموعة من الـframes(8sample/frame وبإيجاد الـDCT لهابل

$\mathrm{Y}(\mathrm{k})=\mathrm{w}(\mathrm{k}) \sum_{\mathrm{n}=1}^{\mathrm{N}} \mathrm{x}(\mathrm{n})\left(\cos \quad \frac{\pi(2 \mathrm{n}-1)(\mathrm{k}-1)}{2 \mathrm{~N}}\right)$ في المعادلة[6]:

Where: $\mathrm{K}=1,2, \ldots \mathrm{N}$

$\mathrm{W}(k)= \begin{cases}\frac{1}{\sqrt{N}}, & k=1 \\ \sqrt{\frac{2}{N}}, & 2 \leq k \leq N\end{cases}$

ثم يتم إخفاء bit واحد في كل frame وعن طريق الاتفاق بين الطرفين يتم تحديد المعاملات التي يتم استخدامها في

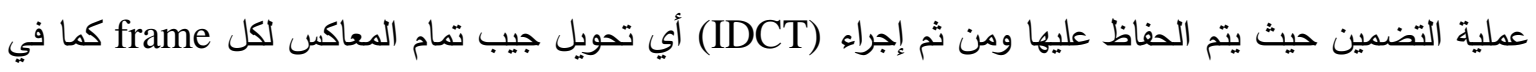
$\mathrm{X}(\mathrm{n})=\sum_{\mathrm{k}=1}^{\mathrm{N}} \mathrm{y}(\mathrm{k}) * \mathrm{w}(\mathrm{k})\left(\cos \quad \frac{\pi(2 \mathrm{n}-1)(\mathrm{k}-1)}{2 \mathrm{~N}}\right)$ المعادلة[7]:

Where $: \mathrm{n}=1,2, \ldots \ldots \mathrm{N}$.
$\mathrm{w}(k)= \begin{cases}\frac{1}{\sqrt{N}}, & k=1 \\ \sqrt{\frac{2}{N}}, & 2 \leq k \leq N\end{cases}$

Where $: \mathrm{n}=1,2, \ldots \ldots \mathrm{N}$.
$\mathrm{w}(k)= \begin{cases}\frac{1}{\sqrt{N}}, & k=1 \\ \sqrt{\frac{2}{N}}, & 2 \leq k \leq N\end{cases}$

تتمثل الخوارزمية المقترحة في إخفاء نص مشفر باستخدام طريقة الـ Direct standerd أو Caesar

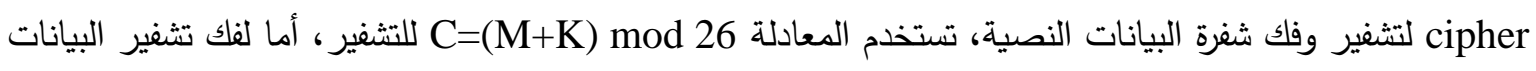

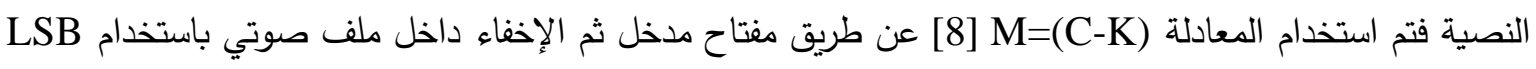

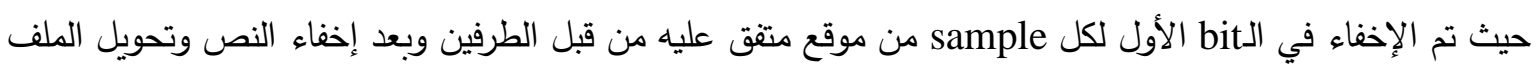
الصوتي الأول إلى سلسلة من الأرقام الثنائية يتم تحويل الملف الصوتي الثاني باستخدام تحويل جيب تمام المتقطع

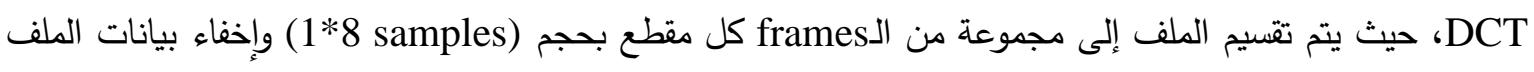
الصوتي الأول بحيث يتم إخفاء bit واحد لكل frames ثم إيجاد IDCT(أي تحويل جيب تمام المتقطع المعاكس) وقد 
تم إخفاء عدد حروف الرسالة وقيمة المفتاح وحجم الملف الصوتي الثاني في مواقع متقق عليها من أجل استرجاع البيانات.

أما خوارزمية الاسترجاع فتتمثل باسترجاع النص بعد عملية فك الثفرة وبصورة صحيحة، إذ يتم استرجاع البيانات من مواقع متفق عليها من قبل الطرفين والمتمثلة بعدد الحروف وحجم ملف الصوتي الأول وقيمة المفتاح

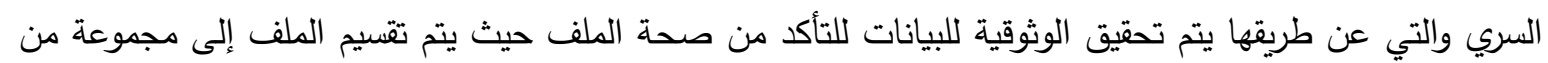

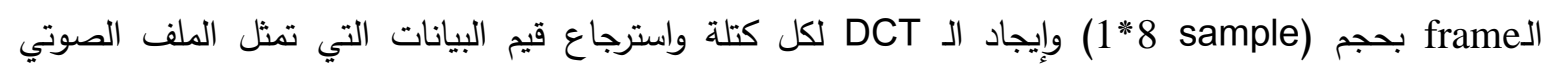

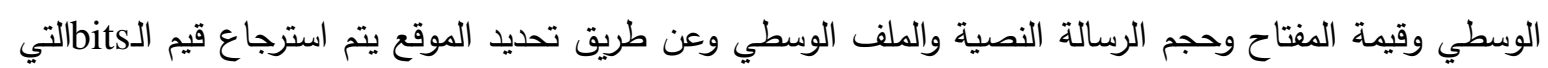
تمثل الرسالة النصية وفك شفرة باستخدام المفتاح المسترجع، يتم استرجاع بيانات النص الأصلي.

\section{3 خوارزمية الإخفاء المقترحة:}

1- قراءة الرسالة النصية.

2- تثفير الرسالة باستخدام مفتاح متفق عليه وتحويلها إلى النظام الثنائي.

3- قراءة الملف الصوتي الأول وإيجاد طول الملف.

4- أ- إذا كان حجم الملف الصوتي أقل من عدد الـ bits للرسالة النصية أطبع الرسالة "لا يمكن استخدام الملف الصوتي الأول في إخفاء بيانات الرسالة المشفرة" أتجه إلى الخطوة (16).

$$
\text { ب- إذا الجواب (لا)، استمر ل... }
$$

5- إخفاء البيانات داخل الملف الصوتي في الخلية الأولى الأقل أهمية.

6- كتابة الناتج في ملف صوتي.

7- حساب قيمة SNR للملف.

8- قراءة الملف الصوتي الثاني وحساب طول الملف.

9- قراءة الملف الصوتي الأول وتحويل إلى النظام الثنائي.

10- أ- إذا كان الملف الصوتي الثاني أكبر من الملف الصوتي الأول الممثل بشكل ثنائي.

ب- إذا كان الجواب لا أطبع الرسالة "لا يمكن استخدام الملف الصوتي الثاني في إخفاء بيانات ملف الصوتي لأني

الأول اتجه إلى الخطوة (16).

11- تقسيم الملف الصوتي إلى مجموعة من الأطر وإيجاد DCT للملف الصوتي الثاني (حجم الـ Frame

(Sample8/

12- إخفاء bit واحد في كل مقطع داخل الملف الثاني.

13- إيجاد IDCT.

14- إخفاء قيمة المفتاح وعدد الحروف للرسالة النصية وحجم الملف الصوتي الأول داخل الملف الصوتي الثاني.

15- حساب قيمة إخعاء فيمة

16- نهاية الخوارزمية

والثكل (1) يوضح المخطط الانسيابي لخوارزمية الإخفاء المقترحة. 


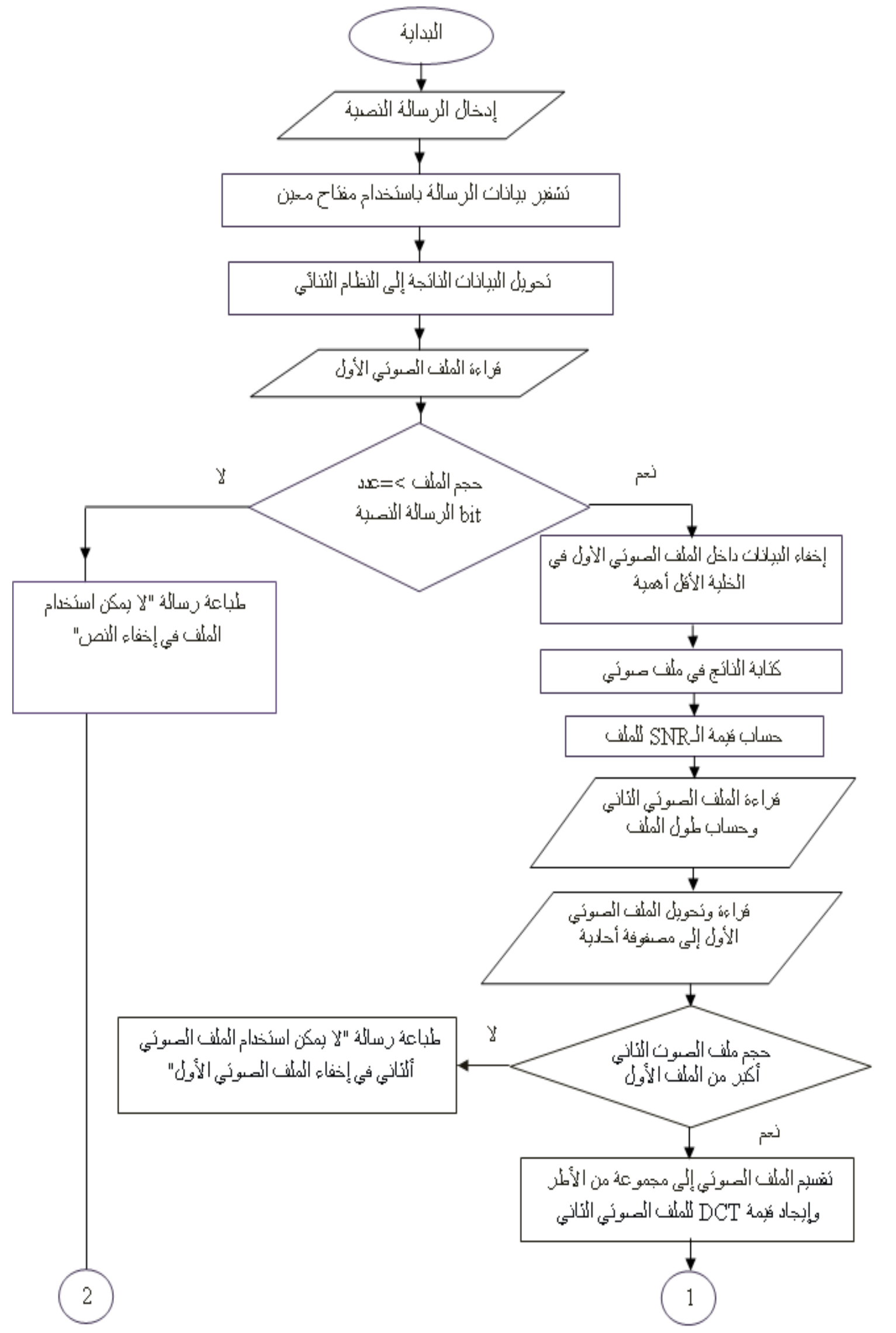




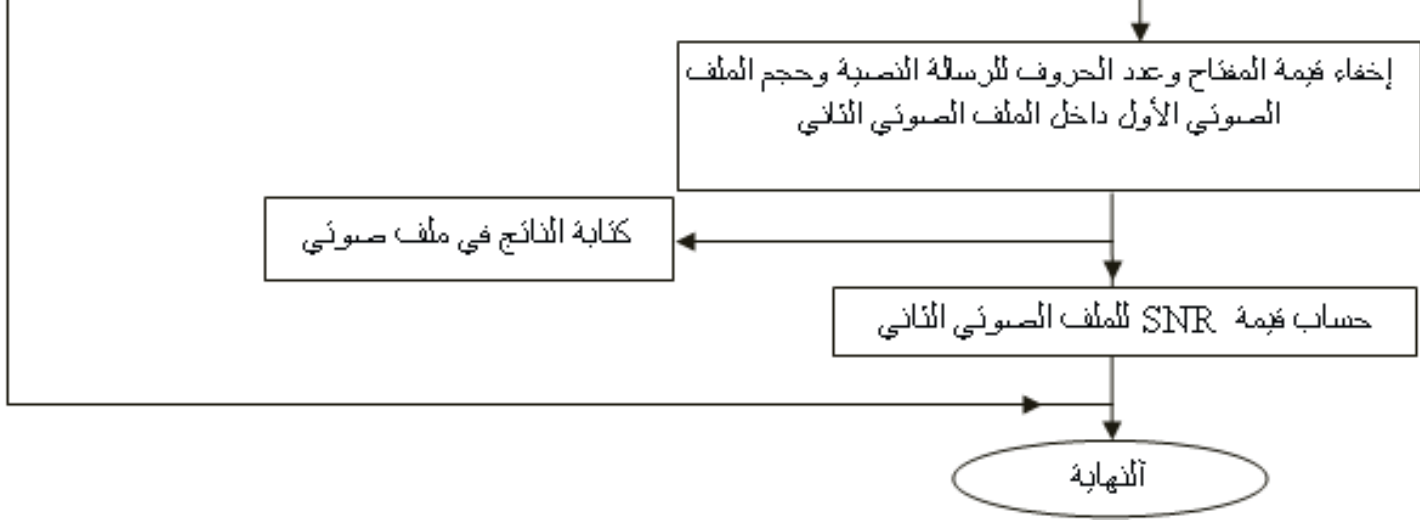

الثكل (1). المخطط الانسيابي لخوارزمية الإخفاء المقترحة

2.3 خوارزمية الاسترجاع المقترحة:

1- قراءة الملف الصوتي الثاني واسترجاع عدد الحروف للرسالة وقيمة المفتاح وحجم الملف الصوتي الأول في المواقع التي تم الاتفاق عليها من قبل الطرفين.

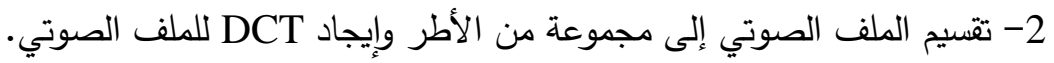
3- استرجاع bit واحد من كل Frame وتحويلها إلى مصنوفة أحادية.

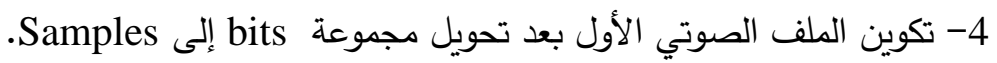
5- حساب حجم الملف الصوتي الأول. 6- أ- إذا كان حجم الملف الصوتي الأول يساوي القيمة ألمسترجعة. ب- إذا كان الجواب لا أطبع الرسالة "الملف المسترجع خاطئ" ثم اتجه إلى الخطوة (11). 7- استرجاع بيانات الرسالة النصية من الملف الصوتي. 8- إجراء عملية فك الثفرة بالاعتماد على قيمة المفتاح المسترجع. 9- أ- إذا كانت عدد الحروف المسترجعة تساوي عدد الحروف المسترجعة من الملف الصوتي الثاني احسب قيمة NC للنص. ب- إذا كان الجواب لا، أطبع الرسالة "الرسالة النصية خاطئة" اتجه إلى الخطوة(11). 10- طباعة الرسالة النصية وقيمة NC لهان.

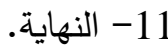
الثكل (2) يوضح المخطط ألانسيابي لعملية الاسترجاع 


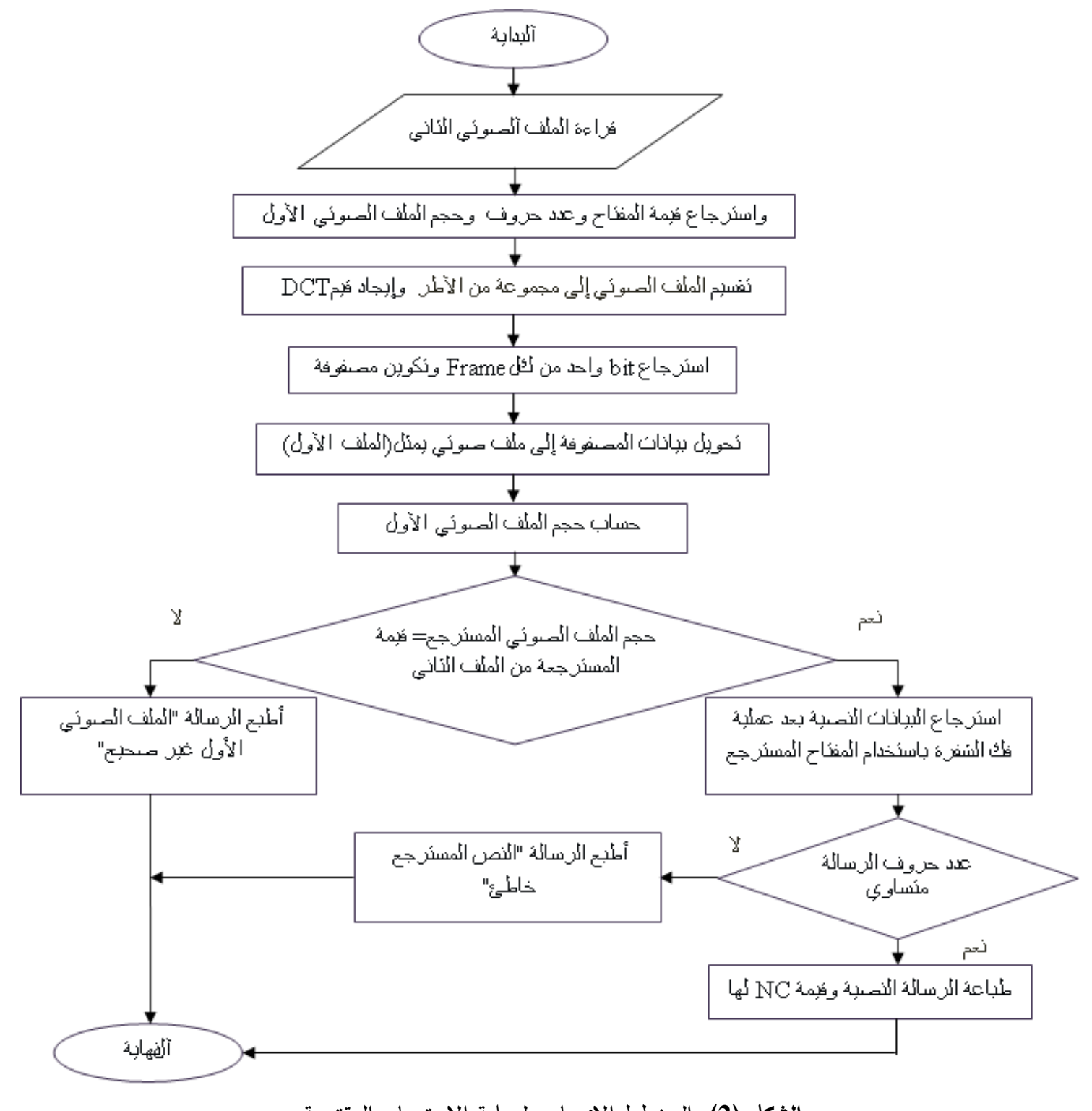

الثكل (2). المخطط الانسيابي لعملية الاسترجاع المقترحة

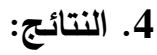

بعد تطبيق خطوات الخوارزمية الأولى والثانية تم استخدام المقاييس الآتية للتأكد من صحة البيانات المسترجعة وجودة الملفات الصوتية الحاوية على البيانات السرية:

1.4 معامل الارتباط التعياري Normalization Correlation (NC):

للتأكد من دقة البيانات المسترجعة تم اعتماد هذا المقياس والموضح في المعادلة (3)، كانت النتائج بالنسبة [6][9][1]. للصوت الأول وحروف الرسالة النصية تساوي واحد (3C=1) $\mathrm{NC}=\sum \mathrm{i} \operatorname{sw}(\mathrm{i}) * \mathrm{~s}(\mathrm{i}) / \sum \mathrm{i}(\mathrm{s}(\mathrm{i}))^{\wedge} 2$

2.4 مقياس نسبة الإثشارة إلى الضوضاء Signal to Noise Ratio (SNR) تم استخدام هذا المقياس للإثشارة إلى عدم إدراك بيانات الرسالة السرية داخل الإشارة الأصلية وعدم ملاحظتها

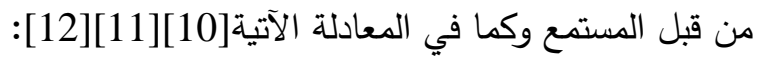


$\mathrm{SNR}=10 * \log _{10} \frac{\sum_{n=0}^{N-1} X^{2}(n)}{\sum_{n=0}^{N-1}\left[X^{\circ}(n)-X(n)\right]^{2}}$

حيث أن: X(n) تمثل الإشارة الأصلية. و و X الإثارة الحاوية على العلامة المائية.

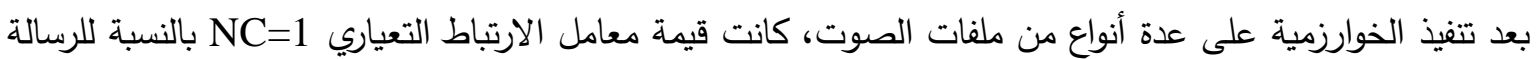

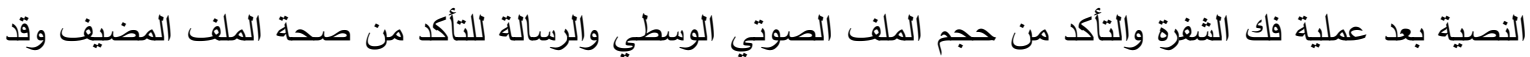
كانت النتائج كما في الجدول التالي:

الجدول (1). يبين نتائج الخوارزمية

\begin{tabular}{|c|c|c|c|c|c|}
\hline $\begin{array}{c}\text { SNR } \\
\text { للملف الصوتي }\end{array}$ & $\begin{array}{c}\text { NC } \\
\text { لحروف الرسالة }\end{array}$ & $\begin{array}{c}\text { NC } \\
\text { للصوتطي }\end{array}$ & النصية الزية & الملف الصوتي & الملف الصوتي \\
\hline 31.7136 & 1 & 1 & $\begin{array}{c}\text { University Of Mosul } \\
\text { (19char) }\end{array}$ & $\begin{array}{c}\text { paving.wav } \\
\text { 392KB }\end{array}$ & $\begin{array}{l}\text { ding.au } \\
7.44 \mathrm{~KB}\end{array}$ \\
\hline 33.8847 & 1 & 1 & $\begin{array}{c}\text { Computer Science } \\
\text { (16 char) }\end{array}$ & $\begin{array}{c}\text { wav48.wav } \\
840 \mathrm{~KB} \\
\end{array}$ & $\begin{array}{l}\text { ding.au } \\
7.44 \mathrm{~KB} \\
\end{array}$ \\
\hline 40.5666 & 1 & 1 & $\begin{array}{l}\text { hello world } \\
\text { (11 char) }\end{array}$ & $\begin{array}{c}\text { Fire_new.wav } \\
192 \mathrm{~KB}\end{array}$ & $\begin{array}{l}\text { ding.au } \\
\text { 7.44KB }\end{array}$ \\
\hline
\end{tabular}

5. واجهات التطبيق:

تم الاعتماد في تطبيق الواجهات الرسومية من خلال لغة (Matlab) تحديدا من شاشة (Command) أو (M_file)، فالواجهة الأولى (الرئيسية) تضم فتح ملف صوتي من نوع (audio) وإدخال المفتاح لتشفير الرسالة

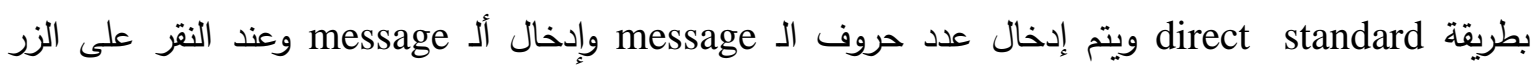

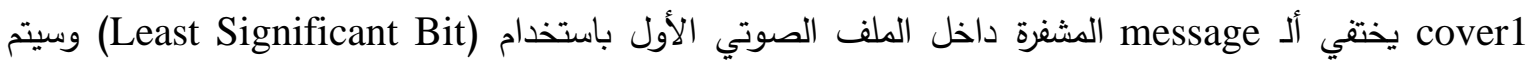

حساب قيمة Signal To Noise Ratio بين الملف الصوتي الأصلي والمضمن وكما مبين في الثكل رقم (3).

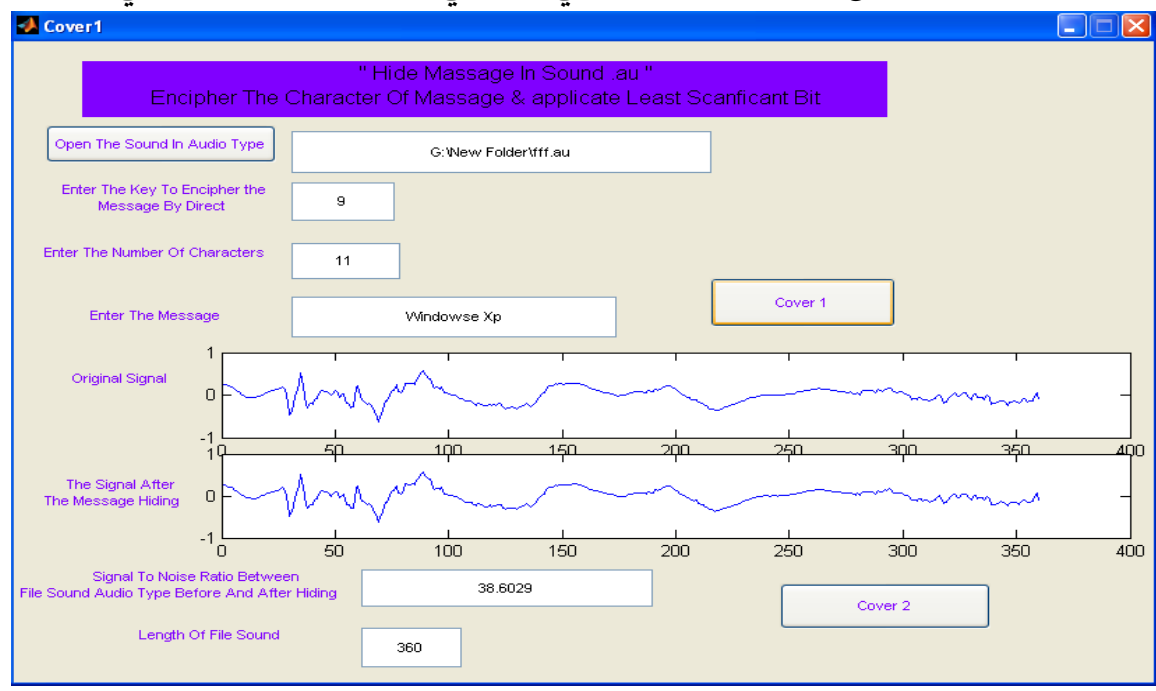

الثكل (3). الواجهة الرئيسية للتطبيق 
وعند النقر على الزر Cover2 الموجود في الواجهة الأولى (الرئيسية) يتم فتح الواجهة الثانية المبينة في الثكل رقم (4) التي يتم فيها فتح الملف الصوتي الثاني (غطاء ثاني للملف الصوتي الأول) المخفي داخله ألـ message بطريقة (DCT) وسيتم حساب قيمة Signal To Noise Ratio بين الملف الصوتي الأصلي الثاني والمضمن.

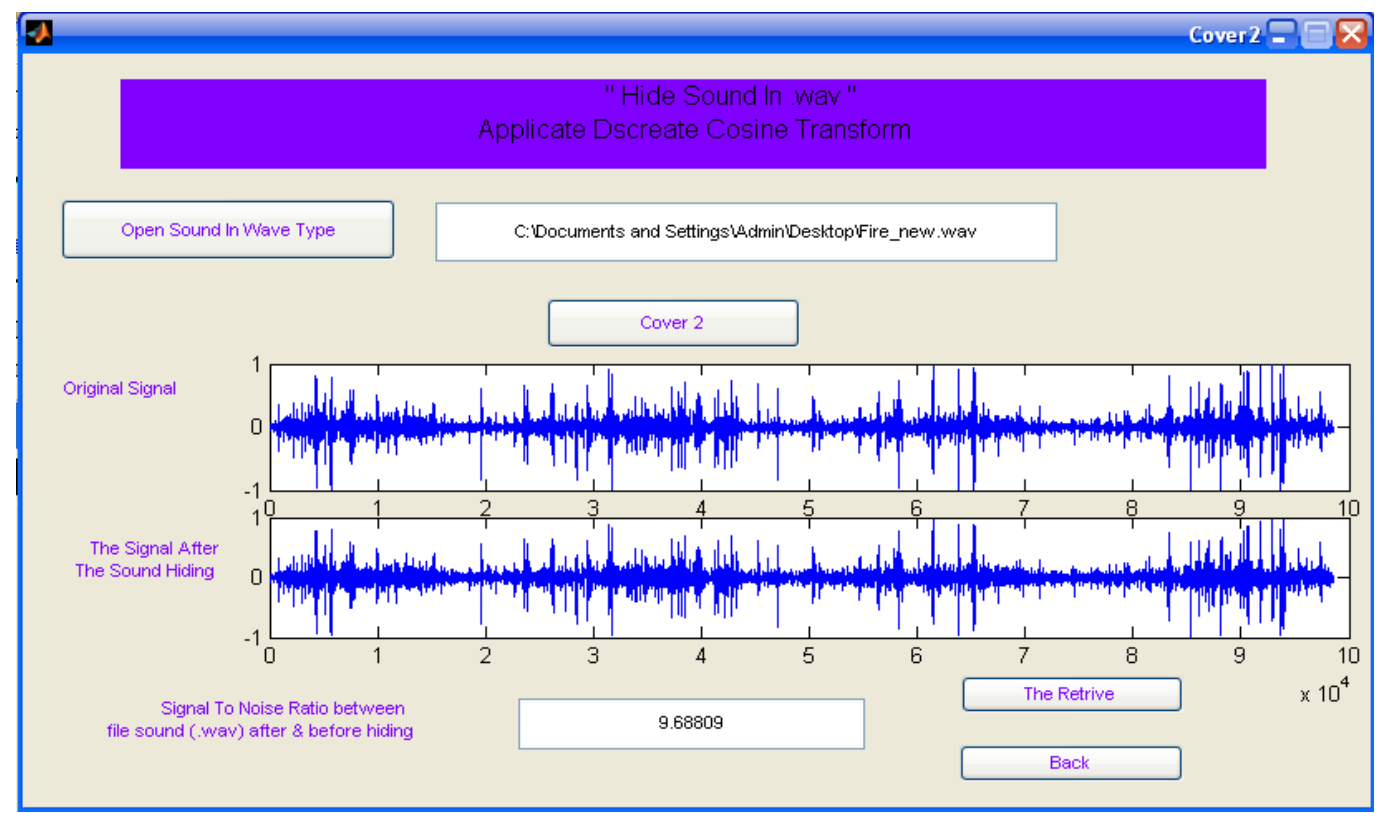

الثكل (4). واجهة الإخفاء الثاني

وعند النقر على الزر retrievethe الموجود في الواجهة الثانية سوف ينتقل إلى الواجهة الثالثة (الاسترجاع)

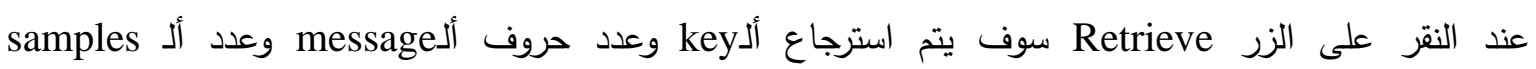
وأل message والملف الصوتي الأول وكما مبين في الثكل رقم (5).

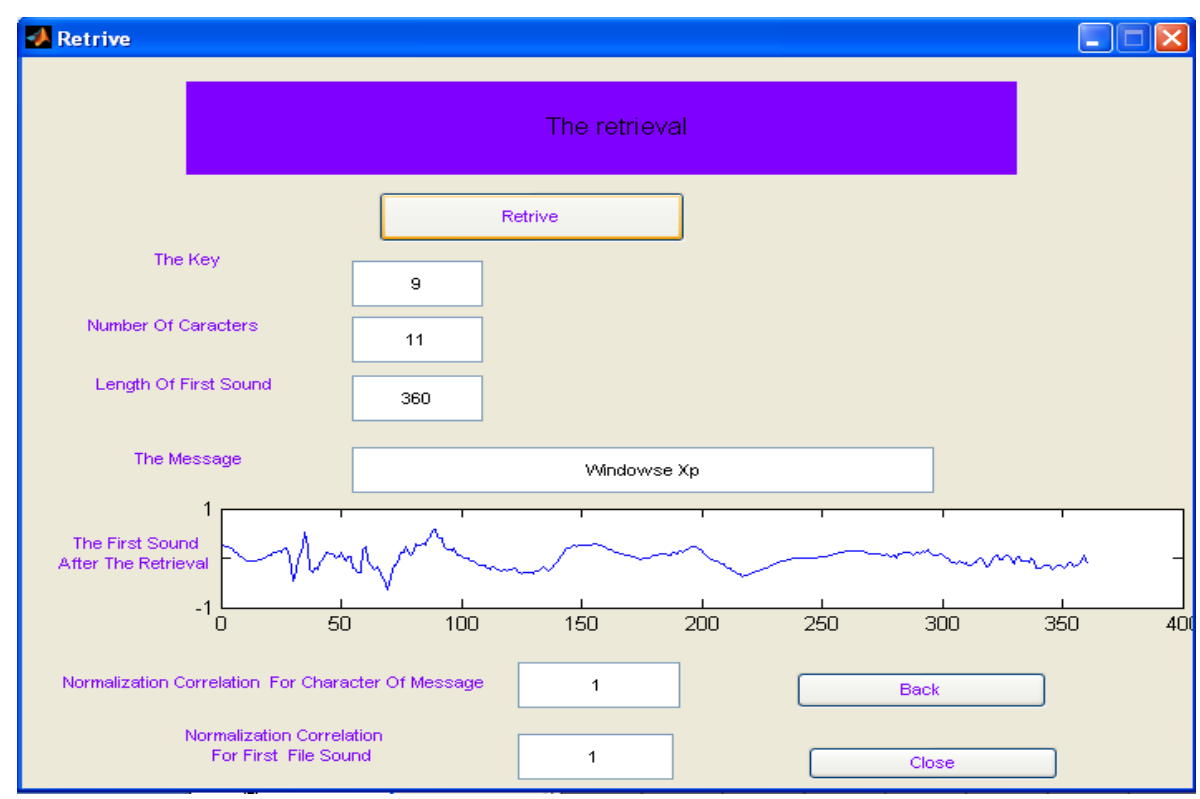

الثكل (5). واجهة الاسترجاع 
بعد تطبيق الخوارزمية المقدمة تم التوصل إلى الاستتتاجات التالية:

1- عملية تطبيق التشفير قبل إخفاء أي ملف أضافت سرية عالية للبيانات حيث لا يمكن الوصول إلى التى النص

الصريح حتى لو حصل شك في وجود بيانات مخفية.

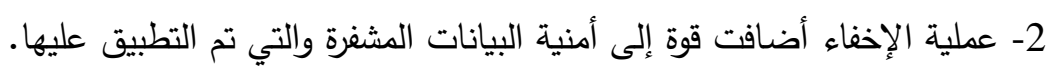

3- بزيادة حجم ملف الصوت المضيف تبين أنه مهما كان حجم النص أو حجم الملف الصوتي الوسطي فإن النتائج

تكون مرضية ولا يتم ظهور أي علامة أو شك في وجود بيانات.

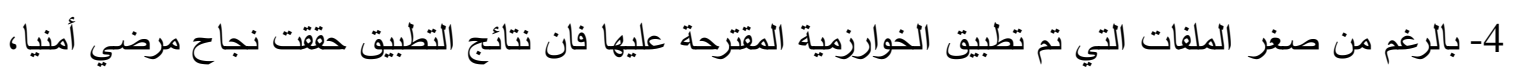

وباختبارها سمعياً لم يظهر أي تغيير على الملف المضيف.

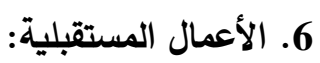

1- تنفيذ البنية المادية المعمارية لخوارزمية الإخفاء والاسترجاع على رقاقة الـ FPGA.

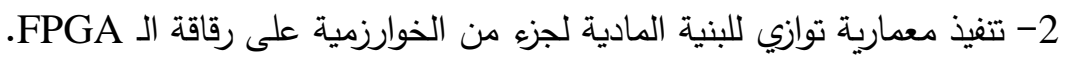

3- تطبيق فكرة ألخوارزمية على أنواع أخرى من ملفات ألوسائط ألمتعددة. 


\section{المصاد}

الصميدعي، عامر تحسين سهيل، 2002، "تطبيق نظام التغطية"، بحث ماجستير، قسم علوم الحاسوب، كلية

علوم الحاسوب والرياضيات، جامعة الموصل، العراق.

[2] Hong Doo Gun, Park Se Hyoung, Shin Jaeho, 2002, "A Public Key Audio Watermarking Using Patch work Algorithm", Department of Electronic Engineering, Dongguk University, Seoul, 100-715, Korea, proceedings of ITCCSCC.

[3] Jixin Lui, Zherning Lu, 2009, "A multipurpose audio watermarking algorithm based on vector quantization in DCT domain", world academy of science, engineering and technology pp618.

[4] Nidhi H Divecha, N N Jani, 2012, "Image Watermarking Algorithm using DCT, DWT and SVD", IJCA Proceedings on National Conference on Innovative Paradigms in Engineering and Technology (NCIPET 2012) ncipet(10):13-16, Published by Foundation of Computer Science, New York, USA.

الحمامي، علاء حسين، محمد حسين، 2008، "إخفاء المعلومات الكتابة المخفية والعلامة المائية"، مكتبة

$$
\text { جامعة الثارقة ص 29-84-102. }
$$

[6] http://en.wikipedia.org/wiki/Discrete_cosine_transform.

[7] R Sridevi, Dr. A Damodaram, Dr. Svl. Narasimham, 2009, "Efficient method of audio steganography by modified LSB algorithm and strong encryption key with enhanced security", Journal of Theoritical and applied information technology, Jntuh, Hyderabad.

[8] Al wahab Abed Adel, 2010, "Attack of multiplicative inverse without using extended Euclid's algorithm", Diyala Journal for pure sciences, vol. 6, no. 4., Dyala University College of education / AL-Razy Computer science department.

[9] Teruya Minamolo, Kenatro Aoki, 2010, "A blind Digital Image Watermarking Method using interval wavelet decomposition" International journal of signal processing, image processing and Pattern recognition, vol.3, no2, department of information science, Saga university, japan.

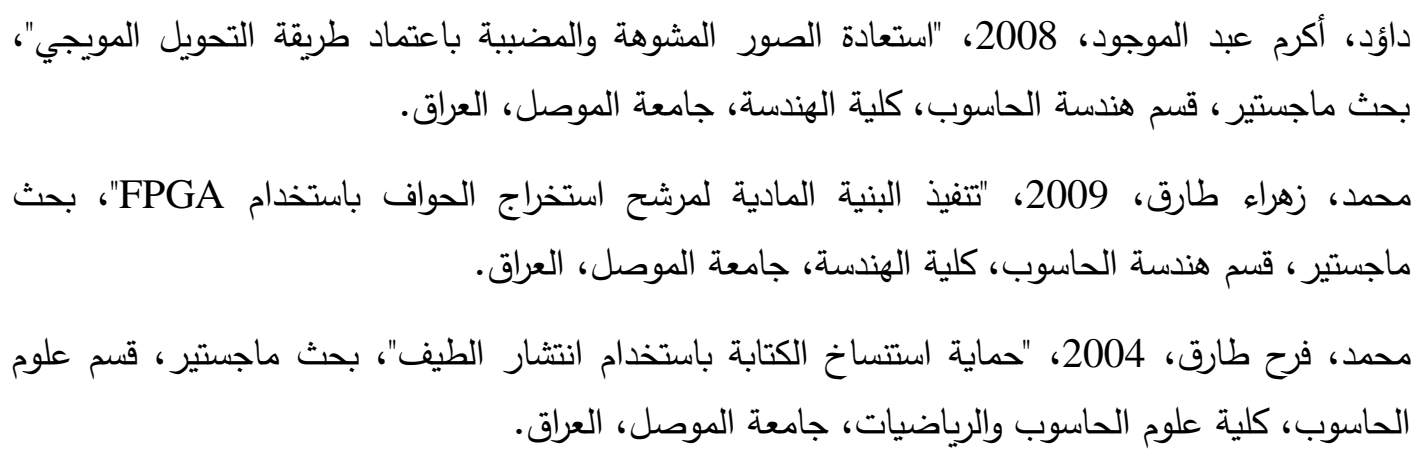

